\title{
SHEAR LOCKING IN A PLANE ELASTICITY PROBLEM AND THE ENHANCED ASSUMED STRAIN METHOD
}

\author{
DIETRICH BRAESS*, PINGBING MING ${ }^{\dagger}$, AND ZHONG-CI SHI ${ }^{\ddagger}$
}

\begin{abstract}
The method of enhanced assumed strains (EAS) is a popular tool for avoiding locking phenomena, e.g., a remedy for shear locking in plane elasticity. We consider bending-dominated problems on thin bodies which can be treated as beams and prove that the degree of approximation of the EAS method is at least as good as that of a beam model. The hypercircle method is combined with arguments of nonconforming methods.
\end{abstract}

Key words. 2D-elasticity, enhanced strain method, shear locking, nonconforming element

AMS subject classifications. 65 N30

1. Introduction. It is well-known that lower-order quadrilateral elements suffer from the following two drawbacks: (a) they lead to shear locking when practicable meshes are used on thin domains in the solution of bending-dominated problems; and (b) volume locking is encountered for nearly incompressible materials. Introduced by Simo AND RifAI [24], the enhanced strain elements (EAS method, for short) are designed to overcome these two shortcomings. They exhibit remarkable improvements over the standard quadrilaterals as extensive numerical tests have shown; see, e.g., [24, 22, 23]. Braess, CARstensen and Reddy [8] have proved that the enhanced element schemes are locking-free in the incompressible limit; we also refer to [8] for a review of the earlier endeavors and to [12] for recent progress in this direction.

Standard quadrilateral elements often lead to spurious shear strain when bendingdominated problems on thin domains are treated in the framework of plane elasticity. Such phenomenon is usually called shear locking. Shear locking has been extensively discussed by MACNEAL in $[14,15]$ from the mechanics aspect of view. PitKäranta [18] has investigated shear locking for the Turner rectangle [29].

The present paper is concerned with a different approach. The danger of shear locking is extreme on thin bodies which can be dealt as beams. We show that the order of convergence for the EAS method is at least as good as a beam model with quadratic terms in the transverse displacement. The beam model is motivated by Morgenstern's analysis of the Kirchhoff plate by the hypercircle method [17], and the dimension reduction is justified. In this way, we obtain convergence in the thin beam limit for the bending-dominated problem. The convergence rate is independent of the element aspect ratio. Of course, elements with high aspect ratio arise in engineering computations of thin bodies like composite beams or plates.

The paper is organized as follows. We will introduce the plane linearized elasticity

\footnotetext{
*Faculty of Mathematics, Ruhr University, 44780 Bochum, Germany (Dietrich.Braess@ruhr-uni-bochum.de)

${ }^{\dagger}$ LSEC, Institute of Computational Mathematics and Scientific/Engineering Computing, AMSS, Chinese Academy of Sciences, No.55. Zhong-Guan-Cun East Road, Beijing, 100190, China (mpb@lsec.cc.ac.cn). The second author was supported by National Natural Science Foundation of China under the grant 10571172, 10871197 and the National Basic Research Program under the grant 2005CB321704

${ }^{\ddagger}$ LSEC, Institute of Computational Mathematics and Scientific/Engineering Computing, AMSS, Chinese Academy of Sciences, No.55. Zhong-Guan-Cun East Road, Beijing, 100190, China (shi@lsec.cc.ac.cn). The third author was supported by National Natural Science Foundation of China under the grant 10571172 and the National Basic Research Program under the grant 2005CB321701
} 
problem and the enhanced strain elements in Section 2. The method can be reformulated as a minimization of a reduced energy [7, Ch. III, §5]. It is shown in Section 3 that the reduced energy is similar to that for the Turner rectangle as discussed by PitKÄranta [18]. An error bound consists of the model error and the constrained interpolation error that will be estimated in Section 4 and Section 5, respectively. In particular, the hypercircle method is combined with arguments of nonconforming methods. Finally we show in an Appendix how sensitive the analysis reacts on the choice of the reduced energy.

Throughout this paper, we assume that the generic constants $C$ and $c$ are independent of the thickness parameter $t$ and the meshsize $h$.

\section{Enhanced Strain Finite Element for Linearized Elasticity Problem.}

2.1. The linearized plane elasticity problem. We consider an isotropic, homogeneous, linearly elastic strip of length $L$ and width $d$ with $d \ll L$. Let $L=1$ and the center of the strip be the origin in 2-space. The strip occupies the region $P_{t}:=P \times I_{t}$, with $P=(-1 / 2,1 / 2), I_{t}=(-t / 2, t / 2)$. The dimensionless parameter $t=d / L$ satisfies $0<t \ll 1$, and this is our main concern throughout the paper. We denote the union of the top and bottom surfaces of the strip by $\partial P_{t}^{ \pm}=P \times\{-t / 2, t / 2\}$ and the lateral boundary by $\partial P_{t}^{\mathrm{L}}=\partial P \times(-t / 2, t / 2)$. We suppose that the strip is loaded by a surface force density $\boldsymbol{g}^{t}: \partial P_{t}^{ \pm} \rightarrow \mathbb{R}^{2}$, and there is no volume force. Moreover, the strip is clamped along its lateral boundary.

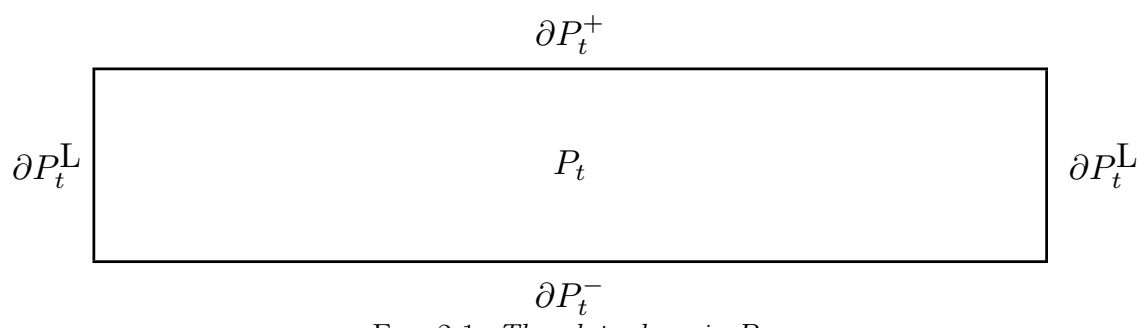

FIG. 2.1. The plate domain $P_{t}$

The displacement $\boldsymbol{u}: P_{t} \rightarrow \mathbb{R}^{2}$ satisfies the boundary-value problems:

$$
\left\{\begin{aligned}
-\operatorname{div} \mathbb{C} \boldsymbol{\epsilon}(\boldsymbol{u}) & =\mathbf{0} & & \text { in } P_{t}, \\
{[[\mathbb{C} \boldsymbol{\epsilon}(\boldsymbol{u}) \boldsymbol{n}]] } & =\boldsymbol{g}^{t} & & \text { on } \partial P_{t}^{ \pm}, \\
\boldsymbol{u} & =\mathbf{0} & & \text { on } \partial P_{t}^{\mathrm{L}},
\end{aligned}\right.
$$

where the infinitesimal strain tensor $\epsilon$ is the symmetric part of the deformation gradient

$$
\boldsymbol{\epsilon}(\boldsymbol{u}):=\frac{1}{2}\left(\nabla \boldsymbol{u}+[\nabla \boldsymbol{u}]^{T}\right) .
$$

The constitutive is given by the fourth-order elasticity tensor $\mathbb{C}$. Specifically,

$$
\mathbb{C} \boldsymbol{\epsilon}=2 \mu \boldsymbol{\epsilon}+\lambda \operatorname{tr}(\boldsymbol{\epsilon}) \mathbf{1},
$$

where $\mathbf{1}$ is the identity, and the Lamé constants $\lambda, \mu$ are related to Young's modulus $E$ and the Poisson ratio $\nu$ by

$$
\lambda=\left\{\begin{array}{ll}
\frac{E \nu}{1-\nu^{2}} & \text { plane stress } \\
\frac{E \nu}{(1+\nu)(1-2 \nu)} & \text { plane strain }
\end{array} \quad \text { and } \quad \mu=\frac{E}{2(1+\nu)} .\right.
$$


Usually $0 \leq \nu<1 / 2$. The flexural rigidity $D$ is defined by

$$
D=\left\{\begin{array}{ll}
\frac{E}{12} E & \text { plane stress, } \\
\frac{E}{12\left(1-\nu^{2}\right)} & \text { plane strain. }
\end{array} \quad \text { and } \quad \gamma= \begin{cases}\nu & \text { plane stress } \\
\frac{\nu}{1-\nu} & \text { plane strain. }\end{cases}\right.
$$

Note that $0 \leq \gamma<1$, and $\gamma \rightarrow 1$ is the incompressible limit, i.e., $\nu \rightarrow 1 / 2$ in the plain strain case.

By the definitions of $\lambda, \mu, D$ and $\gamma$, a straightforward calculation gives the following relations:

$$
\lambda+2 \mu-24 D=\lambda \gamma \quad \text { and } \quad(\lambda+2 \mu) \gamma=\lambda .
$$

It follows from these identities that

$$
24 D=2 \mu(1+\gamma)
$$

As a direct consequence of $(2.3)$ and (2.4), we have for any $\boldsymbol{v}=(u, v) \in\left[H^{1}\left(P_{t}\right)\right]^{2}$ :

$$
\begin{aligned}
\int_{P_{t}} \mathbb{C} \boldsymbol{\epsilon}(\boldsymbol{v}): \boldsymbol{\epsilon}(\boldsymbol{v}) \mathrm{d} \boldsymbol{x}= & 2 \mu\left(\left\|\partial_{x} u\right\|_{L^{2}\left(P_{t}\right)}^{2}+\left\|\partial_{y} v\right\|_{L^{2}\left(P_{t}\right)}^{2}\right) \\
& +\lambda\left\|\partial_{x} u+\partial_{y} v\right\|_{L^{2}\left(P_{t}\right)}^{2}+\mu\left\|\partial_{y} u+\partial_{x} v\right\|_{L^{2}\left(P_{t}\right)}^{2} \\
= & 24 D\left\|\partial_{x} u\right\|_{L^{2}\left(P_{t}\right)}^{2}+\frac{\lambda}{\gamma}\left\|\gamma \partial_{x} u+\partial_{y} v\right\|_{L^{2}\left(P_{t}\right)}^{2} \\
& +\mu\left\|\partial_{y} u+\partial_{x} v\right\|_{L^{2}\left(P_{t}\right)}^{2} .
\end{aligned}
$$

We only consider the bending-dominated plane elasticity problem. Therefore, we assume that the surface traction takes the following form:

$$
\boldsymbol{g}^{t}=\left(g_{1}, g_{2}\right) \text { with } \quad g_{1}=0 \text { and } g_{2}(x, t / 2)=g_{2}(x,-t / 2)=t g(x) .
$$

The space $L^{2}\left(P_{t}\right)$ of square-integrable functions on the domain $P_{t}$ is equipped with the inner product $\langle\cdot, \cdot\rangle$ and the norm $\|\cdot\|_{L^{2}\left(P_{t}\right)}$. Let $H^{m}\left(P_{t}\right)$ denote the standard Sobolev space and

$$
\|v\|_{H^{m}\left(P_{t}\right)}^{2}=\sum_{k=0}^{m}|v|_{H^{k}\left(P_{t}\right)}^{2} \quad \text { and } \quad|v|_{H^{k}\left(P_{t}\right)}^{2}=\int_{P_{t}} \sum_{|\boldsymbol{\alpha}|=k}\left|D^{\boldsymbol{\alpha}} v\right|^{2} \mathrm{~d} \boldsymbol{x} .
$$

Here $\boldsymbol{\alpha}=\left(\alpha_{1}, \alpha_{2}\right)$ is a multi-index whose components $\alpha_{i}$ are non-negative integers, $|\boldsymbol{\alpha}|=\alpha_{1}+\alpha_{2}$, and $D^{\boldsymbol{\alpha}}=\partial^{|\boldsymbol{\alpha}|} / \partial x_{1}^{\alpha_{1}} x_{2}^{\alpha_{2}}$. Define

$$
\boldsymbol{V}:=\left\{\boldsymbol{v} \in\left[H^{1}\left(P_{t}\right)\right]^{2} \mid \boldsymbol{v}=0 \text { on } \partial P_{t}^{\mathrm{L}}\right\}, \quad\|\boldsymbol{\epsilon}(\boldsymbol{v})\|_{\mathbb{C}}:=\langle\mathbb{C} \boldsymbol{\epsilon}(\boldsymbol{v}), \boldsymbol{\epsilon}(\boldsymbol{v})\rangle^{1 / 2}
$$

for any $\boldsymbol{v} \in \boldsymbol{V}$.

The variational problem can be formulated as: find $\boldsymbol{u} \in \boldsymbol{V}$ such that

$$
\int_{P_{t}} \mathbb{C} \boldsymbol{\epsilon}(\boldsymbol{u}): \boldsymbol{\epsilon}(\boldsymbol{v}) \mathrm{d} \boldsymbol{x}=\int_{\partial P_{t}^{ \pm}} \boldsymbol{g}^{t} \cdot \boldsymbol{v} \mathrm{d} s \quad \text { for all } \boldsymbol{v} \in \boldsymbol{V} .
$$


2.2. Enhanced strain finite element approximation. Let $\mathcal{T}_{h}$ be a regular triangulation of $P_{t}$ into rectangles. For any element $T \in \mathcal{T}_{h}$ with the parameter $h:=$ $\max _{T \in \mathcal{T}_{h}} \max \left(h_{T, x}, h_{T, y}\right)$ we let $T:=I_{x} \otimes I_{y}$ with $I_{x}:=\left(x_{T}-h_{T, x} / 2, x_{T}+h_{T, x} / 2\right)$ and $I_{y}:=\left(y_{T}-h_{T, y} / 2, y_{T}+h_{T, y} / 2\right)$. Define $h_{x}:=\max _{T \in \mathcal{T}_{h}} h_{T, x}$ and $h_{y}:=\max _{T \in \mathcal{T}_{h}} h_{T, y}$. Let $\rho$ be the element shape parameter such that $1 \leq h_{T, x} / h_{T, y} \leq \rho$. It is our aim to achieve $\rho$-independent results.

Let $\mathcal{Q}_{1}(T)$ be the standard space of bilinear polynomials on $T$, and set

$$
\boldsymbol{V}^{h}:=\left\{\boldsymbol{v} \in\left[C\left(\bar{P}_{t}\right)\right]^{2} \mid \boldsymbol{v}=0 \text { on } \partial P_{t}^{\mathrm{L}},\left.\boldsymbol{v}\right|_{T} \in \mathcal{Q}_{1}(T) \text { for all } T \in \mathcal{T}_{h}\right\} .
$$

The strain tensor $\boldsymbol{\epsilon}$ is regarded as an independent variable in the framework of the EAS method. In addition to the symmetric gradient of the displacement (2.2) there are enhanced strains. The finite element spaces $\widetilde{\boldsymbol{E}}^{h}$ for enhanced strains are subspaces of the space $\Lambda$ of symmetric $2 \times 2$ matrix-valued functions in $L^{2}\left(P_{t}\right)$, i.e., in the case of homogeneous bodies:

$$
\widetilde{\boldsymbol{E}}^{h} \subset \Lambda:=\left\{\boldsymbol{\tau} \in L^{2}\left(\Omega ; \mathbb{R}_{\mathrm{sym}}^{2 \times 2}\right) \mid \int_{P_{t}} \mathbb{C} \boldsymbol{\tau}(\boldsymbol{x}) \mathrm{d} \boldsymbol{x}=\mathbf{0}\right\} .
$$

Following [8], we formulate the enhanced strain finite element approximation:

Problem 2.1. Find $\left(\boldsymbol{u}_{h}, \boldsymbol{a}_{h}\right) \in \boldsymbol{V}^{h} \times \widetilde{\boldsymbol{E}}^{h}$ such that, for all $\left(\boldsymbol{v}_{h}, \boldsymbol{b}_{h}\right) \in \boldsymbol{V}^{h} \times \widetilde{\boldsymbol{E}}^{h}$,

$$
\int_{P_{t}} \mathbb{C}\left(\boldsymbol{\epsilon}\left(\boldsymbol{u}_{h}\right)+\boldsymbol{a}_{h}\right):\left(\boldsymbol{\epsilon}\left(\boldsymbol{v}_{h}\right)+\boldsymbol{b}_{h}\right) \mathrm{d} \boldsymbol{x}=\int_{\partial P_{t}^{ \pm}} \boldsymbol{g} \cdot \boldsymbol{v}_{h} \mathrm{~d} s .
$$

There are several choices of $\widetilde{\boldsymbol{E}}^{h}$. We focus on the finite elements suggested by Simo AND RIFAI [24]. It is known to be equivalent to the nonconforming part of the element of TAYLOR, BERESFORD, AND WILsON [25]:

$$
\begin{aligned}
\widetilde{\boldsymbol{E}}^{h} & :=\left\{\boldsymbol{b} \in \Lambda|\boldsymbol{b}|_{T} \in \mathcal{L}(T) \text { for all } T \in \mathcal{T}_{h}\right\} \\
\mathcal{L}(T) & :=\operatorname{span}\left\{\left(\begin{array}{ll}
x & 0 \\
0 & 0
\end{array}\right),\left(\begin{array}{ll}
0 & 0 \\
0 & y
\end{array}\right),\left(\begin{array}{ll}
0 & x \\
x & 0
\end{array}\right),\left(\begin{array}{ll}
0 & y \\
y & 0
\end{array}\right)\right\} .
\end{aligned}
$$

Here $x$ and $y$ are the (local) coordinates in the reference element.

The following strengthened Cauchy inequality is the key to the well-posedness of Problem $2.1[6,7]$. There exists a constant $\kappa(0<\kappa<1)$ that is independent of $\lambda$ and $h$, but depends on $\rho$ such that

$$
|\langle\boldsymbol{\epsilon}(\boldsymbol{v}), \boldsymbol{b}\rangle| \leq \kappa\|\boldsymbol{\epsilon}(\boldsymbol{v})\|_{L^{2}\left(P_{t}\right)}\|\boldsymbol{b}\|_{L^{2}\left(P_{t}\right)} \quad \text { for all } \boldsymbol{v} \in \boldsymbol{V}^{h}, \boldsymbol{b} \in \widetilde{\boldsymbol{E}}^{h} .
$$

The above inequality has been proven in [6] for $\boldsymbol{V}^{h}$ and $\widetilde{\boldsymbol{E}}^{h}$ defined in (2.8) and (2.10), respectively. A similar result may be found in [13]. By pursuing the dependence of $\kappa$ on $\rho$, we have

$$
\kappa=\frac{\rho}{\sqrt{2+\rho^{2}}}
$$

By $\left[6\right.$, Lemma A], the inequality (2.11) yields for all $\boldsymbol{v} \in \boldsymbol{V}^{h}$ and $\boldsymbol{b} \in \widetilde{\boldsymbol{E}}^{h}$ :

$$
\|\boldsymbol{\epsilon}(\boldsymbol{v})+\boldsymbol{b}\|_{L^{2}\left(P_{t}\right)} \geq\left(\frac{1-\kappa}{2}\right)^{1 / 2}\left(\|\boldsymbol{\epsilon}(\boldsymbol{v})\|_{L^{2}\left(P_{t}\right)}+\|\boldsymbol{b}\|_{L^{2}\left(P_{t}\right)}\right) .
$$


Based on (2.13) (or equivalently on (2.11)), and under the assumption that the triangulation $\mathcal{T}_{h}$ is a refinement of $\mathcal{T}_{2 h}$, BRAEss, CARSTEnsen, AND REDDY [8] proved the following result showing that the finite elements with enhanced assumed strains are free of volumetric locking.

THEOREM 2.2. There exits a constant $c$ independent of $\lambda, \mathcal{T}_{h}$, and $\boldsymbol{u}$ such that

$$
\left\|\boldsymbol{u}-\boldsymbol{u}_{h}\right\|_{H^{1}\left(P_{t}\right)}+\left\|\boldsymbol{a}_{h}\right\|_{L^{2}\left(P_{t}\right)} \leq \operatorname{ch}\left\|\boldsymbol{g}^{t}\right\|_{H^{3 / 2}\left(\partial P_{t}^{ \pm}\right)} .
$$

It is clear that the right-hand side of (2.14) depends on the size of the domain $P_{t}$. A scaling argument gives that the constant $c$ is at least $\mathcal{O}\left(t^{-2}\right)$, which does not guarantee the uniform convergence of the enhanced assumed strain finite element method in the limit $t \rightarrow 0$. However, Theorem 5.4 below will show that the enhanced strain scheme is actually free of shear locking, as suggested by the extensive numerical examples in Simo AND RifaI [24] and in [22, 23]. The next three sections are devoted to the proof of this result.

3. Error Estimate for Finite Elements with Enhanced Strains. Following PITKÄRANTA [18] we start with the characterization of a special norm that is related to the reduced energy which is induced by the enhanced strains. We will estimate the error with respect to this special norm.

Denote the $L^{2}$ projection onto the piecewise constant functions by $\Pi_{0}$. For any $v$, we have

$$
\left.\left(\Pi_{0} v\right)\right|_{T}=f_{T} v(\boldsymbol{x}) \mathrm{d} \boldsymbol{x} .
$$

Moreover, we define the $L^{2}$ projections in the $x$ and the $y$ direction, respectively:

$$
\Pi_{x} v=f_{I_{x}} v(x, y) \mathrm{d} x, \quad \Pi_{y} v=f_{I_{y}} v(x, y) \mathrm{d} y .
$$

Recalling (2.9) we rewrite Problem 2.1 as a minimization problem:

$$
\left(\boldsymbol{u}_{h}, \boldsymbol{a}_{h}\right)=\underset{(\boldsymbol{v}, \boldsymbol{b}) \in \boldsymbol{V}^{h} \times \widetilde{\boldsymbol{E}}^{h}}{\operatorname{argmin}}\left\{\frac{1}{2} \int_{P_{t}} \mathbb{C}(\boldsymbol{\epsilon}(\boldsymbol{v})+\boldsymbol{b}):(\boldsymbol{\epsilon}(\boldsymbol{v})+\boldsymbol{b}) \mathrm{d} \boldsymbol{x}-\int_{\partial P_{t}^{ \pm}} \boldsymbol{g}^{t} \cdot \boldsymbol{v} \mathrm{d} s\right\} .
$$

The problem above can be viewed as a two-stage minimization problem, namely first with respect to $\boldsymbol{b}$ and then to $\boldsymbol{v}$. The result of the first step is described by the following theorem.

ThEOREM 3.1. For $\boldsymbol{v}=(u, v) \in \boldsymbol{V}^{h}$, we have

$$
\min _{\boldsymbol{b} \in \widetilde{\boldsymbol{E}}^{h}}\langle\mathbb{C}(\boldsymbol{\epsilon}(\boldsymbol{v})+\boldsymbol{b}), \boldsymbol{\epsilon}(\boldsymbol{v})+\boldsymbol{b}\rangle=\|\boldsymbol{v}\|^{2},
$$

where

$$
\begin{aligned}
\|\boldsymbol{v}\|^{2}:= & 24 D\left\|\partial_{x} u\right\|_{L^{2}\left(P_{t}\right)}^{2}+\frac{\lambda}{\gamma}\left\|\Pi_{0}\left(\gamma \partial_{x} u+\partial_{y} v\right)\right\|_{L^{2}\left(P_{t}\right)}^{2} \\
& +\mu\left\|\Pi_{0}\left(\partial_{y} u+\partial_{x} v\right)\right\|_{L^{2}\left(P_{t}\right)}^{2}+24 D\left\|\left(I-\Pi_{x}\right) \partial_{y} v\right\|_{L^{2}\left(P_{t}\right)}^{2} \quad \text { for all } \boldsymbol{v} \in \boldsymbol{V} .
\end{aligned}
$$


Remark. We note that

$$
\Pi_{0}\left(\partial_{y} v\right)=\Pi_{x} \Pi_{y}\left(\partial_{y} v\right)=\Pi_{x}\left(\partial_{y} v\right) \quad \text { for all } \boldsymbol{v} \in \boldsymbol{V}^{h} .
$$

The error bound for the EAS method will refer to the norm (3.2) and to the projector $\Pi_{x}$ in its last term in order to cope with possible boundary layers.

Proof. Obviously, it is sufficient to prove (3.1) on each element $T \in \mathcal{T}_{h}$. Given $\boldsymbol{v}=(u, v) \in \boldsymbol{V}^{h}$, it follows from (2.6) that

$$
\begin{aligned}
\int_{T} \mathbb{C}(\boldsymbol{\epsilon}(\boldsymbol{v}) & +\boldsymbol{b}):(\boldsymbol{\epsilon}(\boldsymbol{v})+\boldsymbol{b}) \mathrm{d} \boldsymbol{x}=24 D\left\|\partial_{x} u+b_{11}\right\|_{L^{2}(T)}^{2} \\
& +\frac{\lambda}{\gamma}\left\|\gamma\left(\partial_{x} u+b_{11}\right)+\left(\partial_{y} v+b_{22}\right)\right\|_{L^{2}(T)}^{2}+\mu\left\|\partial_{y} u+\partial_{x} v+2 b_{12}\right\|_{L^{2}(T)}^{2} .
\end{aligned}
$$

The functions in $\widetilde{\boldsymbol{E}}^{h}$ are $L^{2}$ orthogonal to piecewise constants, and we get

$$
\begin{aligned}
& b_{12}=-\frac{1}{2}\left(I-\Pi_{0}\right)\left(\partial_{y} u+\partial_{x} v\right), \\
& b_{22}=-\gamma\left(I-\Pi_{0}\right) \partial_{x} u .
\end{aligned}
$$

Since the original expression (3.1) is symmetrical in $x$ and $y$, we obtain by a symmetry argument from (3.6)

$$
b_{11}=-\gamma\left(I-\Pi_{0}\right) \partial_{y} v
$$

The nontrivial term in (3.4) becomes

$$
\begin{aligned}
\gamma\left(\partial_{x} u+b_{11}\right)+\left(\partial_{y} v+b_{22}\right) & =\gamma\left[\partial_{x} u-\gamma\left(I-\Pi_{0}\right) \partial_{y} v\right]+\partial_{y} v-\gamma\left(I-\Pi_{0}\right) \partial_{x} u \\
& =\Pi_{0}\left(\gamma \partial_{x} u+\partial_{y} v\right)+\left(1-\gamma^{2}\right)\left(I-\Pi_{0}\right) \partial_{y} v .
\end{aligned}
$$

The orthogonality of the terms yields

$$
\begin{aligned}
\left\|\gamma\left(\partial_{x} u+b_{11}\right)+\left(\partial_{y} v+b_{22}\right)\right\|_{L^{2}(T)}^{2}= & \left\|\Pi_{0}\left(\gamma \partial_{x} u+\partial_{y} v\right)\right\|_{L^{2}(T)}^{2} \\
& +\left(1-\gamma^{2}\right)^{2}\left\|\left(I-\Pi_{0}\right) \partial_{y} v\right\|_{L^{2}(T)}^{2} .
\end{aligned}
$$

Similarly, $\left\|\partial_{x} u+b_{11}\right\|_{L^{2}(T)}^{2}=\left\|\partial_{x} u\right\|_{L^{2}(T)}^{2}+\gamma^{2}\left\|\left(I-\Pi_{0}\right) \partial_{y} v\right\|_{L^{2}(T)}^{2}$. It remains to determine the factor of the term $\left\|\left(I-\Pi_{0}\right) \partial_{y} v\right\|_{L^{2}(T)}^{2}$. It follows from (2.3) that $\frac{\lambda}{\gamma}\left(1-\gamma^{2}\right)=\frac{\lambda}{\gamma}-\lambda \gamma=[\lambda+2 \mu]-[\lambda+2 \mu-24 D]=24 D$. Hence,

$$
24 D \gamma^{2}+\frac{\lambda}{\gamma}\left(1-\gamma^{2}\right)^{2}=24 D \gamma^{2}+24 D\left(1-\gamma^{2}\right)=24 D .
$$

Collecting all terms we obtain (3.2), and the proof is complete.

REMARK 3.2. This theorem can be viewed as a reformulation of the static condensation procedure in [27].

Compared to (2.6), the expression $\|\cdot\|^{2}$ resembles the strain energy. In what follows, we shall show that $\|\cdot \cdot\|^{2}$ is equivalent to the strain energy over $\boldsymbol{V}^{h}$.

The relations (2.3) and (2.4) yield for any $\boldsymbol{v}=(u, v) \in \boldsymbol{V}$ :

$$
\begin{aligned}
\frac{\lambda}{\gamma}\left\|\Pi_{0}\left(\gamma \partial_{x} u+\partial_{y} v\right)\right\|_{L^{2}\left(P_{t}\right)}^{2}= & (\lambda+2 \mu-24 D)\left\|\Pi_{0}\left(\partial_{x} u\right)\right\|_{L^{2}\left(P_{t}\right)}^{2} \\
& +2 \lambda\left\langle\Pi_{0}\left(\partial_{x} u\right), \Pi_{0}\left(\partial_{y} v\right)\right\rangle+(\lambda+2 \mu)\left\|\Pi_{0}\left(\partial_{y} v\right)\right\|_{L^{2}\left(P_{t}\right)}^{2} \\
= & (2 \mu-24 D)\left\|\Pi_{0}\left(\partial_{x} u\right)\right\|_{L^{2}\left(P_{t}\right)}^{2}+2 \mu\left\|\Pi_{0}\left(\partial_{y} v\right)\right\|_{L^{2}\left(P_{t}\right)}^{2} \\
& +\lambda\left\|\Pi_{0}\left(\partial_{x} u+\partial_{y} v\right)\right\|_{L^{2}\left(P_{t}\right)}^{2} .
\end{aligned}
$$


Now we rewrite $\|\boldsymbol{v}\| \mid$ into a more symmetric form

$$
\begin{aligned}
\|\boldsymbol{v}\| \|^{2}= & 2 \mu\left(\left\|\Pi_{0}\left(\partial_{x} u\right)\right\|_{L^{2}\left(P_{t}\right)}^{2}+\left\|\Pi_{0}\left(\partial_{y} v\right)\right\|_{L^{2}\left(P_{t}\right)}^{2}\right) \\
& +\lambda\left\|\Pi_{0}\left(\partial_{x} u+\partial_{y} v\right)\right\|_{L^{2}\left(P_{t}\right)}^{2}+\mu\left\|\Pi_{0}\left(\partial_{y} u+\partial_{x} v\right)\right\|_{L^{2}\left(P_{t}\right)}^{2} \\
& +24 D\left(\left\|\left(I-\Pi_{0}\right) \partial_{x} u\right\|_{L^{2}\left(P_{t}\right)}^{2}+\left\|\left(I-\Pi_{x}\right) \partial_{y} v\right\|_{L^{2}\left(P_{t}\right)}^{2}\right) .
\end{aligned}
$$

LEMMA 3.3. For any $\boldsymbol{v}=(u, v) \in \boldsymbol{V}^{h}$, there holds

$$
\sqrt{\frac{1-\gamma}{2(1+\gamma) \rho^{2}}}\|\boldsymbol{\epsilon}(\boldsymbol{v})\|_{\mathbb{C}} \leq\|\boldsymbol{v}\| \leq\|\boldsymbol{\epsilon}(\boldsymbol{v})\|_{\mathbb{C}} .
$$

Proof. From (2.4) we know that $2 \mu \leq 24 D=2 \mu(1+\gamma)$. Therefore, given $\eta \in L^{2}\left(P_{t}\right)$, we have

$$
\begin{aligned}
2 \mu\|\eta\|_{L^{2}\left(P_{t}\right)}^{2} & \leq 24 D\left\|\Pi_{0}(\eta)\right\|_{L^{2}\left(P_{t}\right)}^{2}+2 \mu\left\|\left(I-\Pi_{0}\right) \eta\right\|_{L^{2}\left(P_{t}\right)}^{2} \\
& \leq 2 \mu(1+\gamma)\|\eta\|_{L^{2}\left(P_{t}\right)}^{2} .
\end{aligned}
$$

Starting from the symmetric form (3.8) and recalling (3.3) we apply the inequality above twice and drop the other two terms to obtain

$$
\|\boldsymbol{v}\|^{2} \geq 2 \mu\left(\left\|\partial_{x} u\right\|_{L^{2}\left(P_{t}\right)}^{2}+\left\|\partial_{y} v\right\|_{L^{2}\left(P_{t}\right)}^{2}\right) .
$$

The components of $\boldsymbol{v}=(u, v) \in \boldsymbol{V}^{h}$ are bilinear functions, and a direct calculation gives

$$
\begin{aligned}
\left\|\left(I-\Pi_{0}\right) \partial_{y} u\right\|_{L^{2}\left(P_{t}\right)} & \leq \rho\left\|\left(I-\Pi_{0}\right) \partial_{x} u\right\|_{L^{2}\left(P_{t}\right)}, \\
\left\|\left(I-\Pi_{0}\right) \partial_{x} v\right\|_{L^{2}\left(P_{t}\right)} & \leq\left\|\left(I-\Pi_{x}\right) \partial_{y} v\right\|_{L^{2}\left(P_{t}\right)},
\end{aligned}
$$

which together with the orthogonality of the different contributions implies

$$
\begin{aligned}
24 D\left(\left\|\left(I-\Pi_{0}\right) \partial_{x} u\right\|_{L^{2}\left(P_{t}\right)}^{2}\right. & \left.+\left\|\left(I-\Pi_{x}\right) \partial_{y} v\right\|_{L^{2}\left(P_{t}\right)}^{2}\right) \\
& \geq \frac{24 D}{\rho^{2}}\left\|\left(I-\Pi_{0}\right)\left(\partial_{y} u+\partial_{x} v\right)\right\|_{L^{2}\left(P_{t}\right)}^{2} .
\end{aligned}
$$

Hence,

$$
\begin{aligned}
\|\boldsymbol{v}\|^{2} & \geq \mu\left\|\Pi_{0}\left(\partial_{y} u+\partial_{x} v\right)\right\|_{L^{2}\left(P_{t}\right)}^{2}+\frac{24 D}{\rho^{2}}\left\|\left(I-\Pi_{0}\right)\left(\partial_{y} u+\partial_{x} v\right)\right\|_{L^{2}\left(P_{t}\right)}^{2} \\
& \geq \frac{\mu}{\rho^{2}}\left\|\partial_{y} u+\partial_{x} v\right\|_{L^{2}\left(P_{t}\right)}^{2} .
\end{aligned}
$$

A convex combination of the above inequality and (3.11) leads to

$$
\|\boldsymbol{v}\|\left\|^{2} \geq \mu\left(\left\|\partial_{x} u\right\|_{L^{2}\left(P_{t}\right)}^{2}+\left\|\partial_{y} v\right\|_{L^{2}\left(P_{t}\right)}^{2}\right)+\frac{\mu}{\rho^{2}}\right\| \partial_{y} u+\partial_{x} v \|_{L^{2}\left(P_{t}\right)}^{2} .
$$

Now, the lower bound in (3.9) follows from this inequality and

$$
\|\boldsymbol{v}\|_{\mathbb{C}}^{2} \leq 2(\lambda+\mu)\|\boldsymbol{\epsilon}(\boldsymbol{v})\|_{L^{2}\left(P_{t}\right)}^{2}=(\lambda+2 \mu)(1+\gamma)\|\boldsymbol{\epsilon}(\boldsymbol{v})\|_{L^{2}\left(P_{t}\right)}^{2} .
$$


The upper bound in the assertion is a direct consequence of the definition (3.1).

The lemma asserts that $\||\cdot| \mid$ is equivalent to the strain energy on $\boldsymbol{V}^{h}$. In particular, we will make use of the upper bound that is independent of the shape parameter $\rho$. A similar upper bound with a slightly larger constant is obtained for elements in $\boldsymbol{V}$.

LEMma 3.4. For any $\boldsymbol{v} \in \boldsymbol{V}$, there holds

$$
\|\boldsymbol{v}\| \leq \sqrt{1+\gamma}\|\boldsymbol{\epsilon}(\boldsymbol{v})\|_{\mathbb{C}}
$$

Proof. Since $\left\|\Pi_{0}\left(\partial_{y} v\right)\right\|_{L^{2}\left(P_{t}\right)} \leq\left\|\Pi_{x}\left(\partial_{y} v\right)\right\|_{L^{2}\left(P_{t}\right)}$, we obtain from the symmetric form (3.8) by using (3.10) twice

$$
\begin{aligned}
\|\boldsymbol{v}\| \leq & 2 \mu(1+\gamma)\left\|\partial_{x} u\right\|_{L^{2}\left(P_{t}\right)}^{2}+\lambda\left\|\partial_{x} u+\partial_{y} v\right\|_{L^{2}\left(P_{t}\right)}^{2} \\
& +\mu\left\|\partial_{y} u+\partial_{x} v\right\|_{L^{2}\left(P_{t}\right)}^{2}+2 \mu(1+\gamma)\left\|\partial_{y} v\right\|_{L^{2}\left(P_{t}\right)}^{2}
\end{aligned}
$$

A comparison with (2.5) yields the assertion (3.14).

REMARK 3.5. If we replace $\Pi_{x}$ in the last term of the definition of $\|\boldsymbol{v}\|$ by $\Pi_{0}$, then the modified expression $\|\left.|\cdot|\right|^{2}$ would coincide with a modified strain energy in BELYTSCHKO AND BACHRACH [3]. The latter, in turn, refers to a reformulation of the classical Turner rectangle [29] as suggested by PITKÄRANTA [18, Theorem 5.1].

Definition 3.6. We call $\widehat{\boldsymbol{\sigma}} \in H\left(\mathrm{div}, P_{t}\right)$ a statically admissible stress tensor or an admissible stress tensor for short, if it satisfies

$$
\operatorname{div} \widehat{\boldsymbol{\sigma}}=\mathbf{0} \quad \text { in } P_{t}, \quad \widehat{\boldsymbol{\sigma}} \cdot \boldsymbol{n}=\boldsymbol{g}^{t} \quad \text { on } \partial P_{t}^{ \pm}
$$

Let

$$
\left\langle\mathbb{C}_{h} \boldsymbol{\epsilon}(\boldsymbol{w}), \boldsymbol{\epsilon}(\boldsymbol{w})\right\rangle:=\|\boldsymbol{w}\|^{2}
$$

and $\left\langle\mathbb{C}_{h} \boldsymbol{\epsilon}(\boldsymbol{w}), \boldsymbol{\epsilon}(\boldsymbol{v})\right\rangle$ be the associated bilinear form.

The following error estimate combines ideas of the theorem of BERGER, SCOTT, AND STRAng [4] with the hypercircle method.

LEMMA 3.7. Let $\widehat{\boldsymbol{\sigma}}$ be an admissible stress tensor with $\boldsymbol{g}^{t}=(0, \operatorname{tg}(x))$ and $\hat{\boldsymbol{u}} \in \boldsymbol{V}$. Then,

$$
\begin{aligned}
\left\|\boldsymbol{u}-\boldsymbol{u}_{h}\right\| \mid \leq & \sqrt{1+\gamma}\|\boldsymbol{\epsilon}(\boldsymbol{u}-\hat{\boldsymbol{u}})\|_{\mathbb{C}}+\inf _{\boldsymbol{w} \in \boldsymbol{V}^{h}}\|\hat{\boldsymbol{u}}-\boldsymbol{w}\| \\
& +\sup _{\boldsymbol{v} \in \boldsymbol{V}^{h}} \frac{\left|\left\langle\mathbb{C}_{h} \boldsymbol{\epsilon}(\hat{\boldsymbol{u}})-\widehat{\boldsymbol{\sigma}}, \boldsymbol{\epsilon}(\boldsymbol{v})\right\rangle\right|}{\|\boldsymbol{v}\|}
\end{aligned}
$$

Proof. Let $\tilde{\boldsymbol{u}} \in \boldsymbol{V}^{h}$ be the solution of the auxiliary problem

$$
\left\langle\mathbb{C}_{h} \boldsymbol{\epsilon}(\tilde{\boldsymbol{u}}), \boldsymbol{\epsilon}(\boldsymbol{v})\right\rangle=\left\langle\mathbb{C}_{h} \boldsymbol{\epsilon}(\hat{\boldsymbol{u}}), \boldsymbol{\epsilon}(\boldsymbol{v})\right\rangle \quad \text { for all } \boldsymbol{v} \in \boldsymbol{V}^{h} .
$$

Setting $\boldsymbol{w}:=\tilde{\boldsymbol{u}}-\boldsymbol{u}_{h}$ we obtain

$$
\begin{aligned}
\|\boldsymbol{w}\|^{2} & =\left\langle\mathbb{C}_{h} \boldsymbol{\epsilon}(\tilde{\boldsymbol{u}}), \boldsymbol{\epsilon}(\boldsymbol{w})\right\rangle-\left\langle\mathbb{C}_{h} \boldsymbol{\epsilon}\left(\boldsymbol{u}_{h}\right), \boldsymbol{\epsilon}(\boldsymbol{w})\right\rangle \\
& =\left\langle\mathbb{C}_{h} \boldsymbol{\epsilon}(\hat{\boldsymbol{u}}), \boldsymbol{\epsilon}(\boldsymbol{w})\right\rangle-\langle\mathbb{C} \boldsymbol{\epsilon}(\boldsymbol{u}), \boldsymbol{\epsilon}(\boldsymbol{w})\rangle \\
& =\left\langle\mathbb{C}_{h} \boldsymbol{\epsilon}(\hat{\boldsymbol{u}})-\widehat{\boldsymbol{\sigma}}, \boldsymbol{\epsilon}(\boldsymbol{w})\right\rangle+\langle\widehat{\boldsymbol{\sigma}}-\mathbb{C} \boldsymbol{\epsilon}(\boldsymbol{u}), \boldsymbol{\epsilon}(\boldsymbol{w})\rangle .
\end{aligned}
$$


Integration by parts and the definition of $\widehat{\sigma}$ yield

$$
\begin{aligned}
\langle\widehat{\boldsymbol{\sigma}}-\mathbb{C} \boldsymbol{\epsilon}(\boldsymbol{u}), \boldsymbol{\epsilon}(\boldsymbol{w})\rangle & =-\langle\operatorname{div} \widehat{\boldsymbol{\sigma}}-\operatorname{div} \mathbb{C} \boldsymbol{\epsilon}(\boldsymbol{u}), \boldsymbol{w}\rangle+\int_{\partial P_{t}}(\widehat{\boldsymbol{\sigma}}-\mathbb{C} \boldsymbol{\epsilon}(\boldsymbol{u})) \cdot \boldsymbol{n} \boldsymbol{w} \mathrm{d} s \\
& =0 .
\end{aligned}
$$

Hence,

$$
\left\|\tilde{\boldsymbol{u}}-\boldsymbol{u}_{h}\right\| \mid=\sup _{\boldsymbol{v} \in \boldsymbol{V}^{h}} \frac{\mid\left\langle\left(\mathbb{C}_{h} \boldsymbol{\epsilon}(\hat{\boldsymbol{u}})-\widehat{\boldsymbol{\sigma}}, \boldsymbol{\epsilon}(\boldsymbol{v})\right\rangle\right|}{\|\boldsymbol{v} \mid\|} .
$$

From the triangle inequality and (3.14) it follows that

$$
\begin{aligned}
\left\|\mid \boldsymbol{u}-\boldsymbol{u}_{h}\right\| & \leq\|\boldsymbol{u}-\hat{\boldsymbol{u}}\||+\|\hat{\boldsymbol{u}}-\tilde{\boldsymbol{u}}\||+\left\|\tilde{\boldsymbol{u}}-\boldsymbol{u}_{h} \mid\right\| \\
& \leq \sqrt{1+\gamma}\|\boldsymbol{\epsilon}(\boldsymbol{u}-\hat{\boldsymbol{u}})\|_{\mathbb{C}}+\|\hat{\boldsymbol{u}}-\tilde{\boldsymbol{u}}\|\left|+\left\|\tilde{\boldsymbol{u}}-\boldsymbol{u}_{h}\right\|\right|,
\end{aligned}
$$

which together with the Galerkin orthogonality $\|\hat{\boldsymbol{u}}-\tilde{\boldsymbol{u}}\|\left|=\inf _{\boldsymbol{v} \in \boldsymbol{V}^{h}}\|\hat{\boldsymbol{u}}-\boldsymbol{v}\|\right|$ and (3.16) gives (3.15).

The lemma will be applied to $\hat{\boldsymbol{u}}$ and $\hat{\boldsymbol{\sigma}}$ from a beam model. The first term accounts for how well the beam model approximates the solution of the bendingdominated plane elasticity problem, cf. Theorem 4.4 below. The last two terms reflect the discretization error of the beam model and will be estimated in Section 5 .

4. Model Error Estimate for Beam Approximation. The main objective of this section is to estimate

$$
\frac{\|\epsilon(\boldsymbol{u}-\hat{\boldsymbol{u}})\|_{\mathbb{C}}}{\|\boldsymbol{\epsilon}(\boldsymbol{u})\|_{\mathbb{C}}}
$$

which characterizes the error between the solution of the two-dimensional elasticity problem $\boldsymbol{u}$ and the $(1,2)$-model of the Euler-Bernoulli beam

$$
\hat{\boldsymbol{u}}:=\left(-y \omega^{\prime}(x), \omega(x)+\frac{\gamma}{2} y^{2} \psi(x)\right) .
$$

Specifically, $\omega$ is the main term of the transverse displacement augmented by a correction of second order in $y$. In contrast to the Timoshenko beam [26] with a more general ansatz for the rotation $\theta: \boldsymbol{u}:=\left(y \theta(x), \omega(x)+\frac{\gamma}{2} y^{2} \psi(x)\right)$, the rotation is fixed here in the spirit of the Kirchhoff hypothesis by $\theta=-\omega^{\prime}$.

Here $\omega$ is the solution of the boundary value problem:

$$
\left\{\begin{array}{c}
D t^{2} \omega^{(4)}(x)=g(x) \quad \text { in } P \\
\omega(-1 / 2)=\omega(1 / 2)=\omega^{\prime}(-1 / 2)=\omega^{\prime}(1 / 2)=0 .
\end{array}\right.
$$

and $\psi$ is the solution of

$$
\left\{\begin{aligned}
-t^{2} \psi^{\prime \prime}(x)+A^{2} \psi(x) & =A^{2} \omega^{\prime \prime}(x) \quad \text { in } P, \\
\psi(-1 / 2)=\psi(1 / 2) & =0
\end{aligned}\right.
$$

with $A:=(20 /(1-\gamma))^{1 / 2} \mu$. To this end we shall exploit the following special form of the Prager-Synge hypercircle theorem [19] or [7, p. 148] and recall Definition 3.6. 
THEOREM 4.1. Let $\widehat{\boldsymbol{\sigma}}$ be an admissible stress tensor and $\overline{\boldsymbol{u}} \in \boldsymbol{V}$. Then for the solution $\boldsymbol{u}$ of Problem 2.1,

$$
\langle\mathbb{C} \epsilon(\boldsymbol{u}-\overline{\boldsymbol{u}}), \boldsymbol{\epsilon}(\boldsymbol{u}-\overline{\boldsymbol{u}})\rangle+\left\langle\mathbb{C}^{-1}(\boldsymbol{\sigma}-\widehat{\boldsymbol{\sigma}}), \boldsymbol{\sigma}-\widehat{\boldsymbol{\sigma}}\right\rangle=\left\langle\mathbb{C}^{-1}(\widehat{\boldsymbol{\sigma}}-\mathbb{C} \epsilon(\overline{\boldsymbol{u}})), \widehat{\boldsymbol{\sigma}}-\mathbb{C} \epsilon(\overline{\boldsymbol{u}})\right\rangle .
$$

To exploit Prager-Synge theorem, we construct an admissible stress tensor. MorGENSTEN's treatment of the $(1,1,2)$-model of the Kirchhoff-Love plate [17] (see also [2, $9,11,21])$ motivates the following choice of the admissible stress tensor and to combine it with the Euler-Bernoulli beam model (4.1). Set

$$
\widehat{\boldsymbol{\sigma}}:=\left(\begin{array}{cc}
-24 D y \omega^{\prime \prime} & \text { symm. } \\
24 D\left(y^{2} / 2-t^{2} / 8\right) \omega^{(3)}(x) & 24\left(1 / 8-y^{2} /\left(6 t^{2}\right)\right) y g(x)
\end{array}\right) .
$$

A priori estimates of $\omega$ and $\psi$ will be derived by the inequality (4.5) below, which can be regarded as a special case of the general Gagliardo-Nirenberg inequality in [1]. We include an elementary proof for the reader's convenience.

LEMma 4.2. Let $(a, b)$ be a finite interval and $\phi \in H^{1}(a, b)$. Then

$$
\|\phi\|_{L_{\infty}}:=\max _{a \leq x \leq b}|\phi(x)| \leq\left(\frac{1}{b-a}+2\right)^{1 / 2}\|\phi\|_{L^{2}(a, b)}^{1 / 2}\|\phi\|_{H^{1}(a, b)}^{1 / 2} .
$$

Moreover, if $\phi$ has a zero in $[a, b]$, then

$$
\|\phi\|_{L_{\infty}} \leq \sqrt{2}\|\phi\|_{L^{2}(a, b)}^{1 / 2}\left\|\phi^{\prime}\right\|_{L^{2}(a, b)}^{1 / 2} .
$$

Proof. For any $x, y \in[a, b]$ we have

$$
\begin{aligned}
\phi^{2}(y) & =\phi^{2}(x)+2 \int_{x}^{y} \phi(s) \phi^{\prime}(s) \mathrm{d} s \leq \phi^{2}(x)+2 \int_{a}^{b}\left|\phi(s) \| \phi^{\prime}(s)\right| \mathrm{d} s \\
& \leq \phi^{2}(x)+2\|\phi\|_{L^{2}(a, b)}\left\|\phi^{\prime}\right\|_{L^{2}(a, b)} .
\end{aligned}
$$

Integrating the above inequality with respect to $x$, we get

$$
\begin{aligned}
\phi^{2}(y) & \leq \frac{1}{b-a}\|\phi\|_{L^{2}(a, b)}^{2}+2\|\phi\|_{L^{2}(a, b)}\left\|\phi^{\prime}\right\|_{L^{2}(a, b)} \\
& =\|\phi\|_{L^{2}(a, b)}\left(\frac{1}{b-a}\|\phi\|_{L^{2}(a, b)}+2\left\|\phi^{\prime}\right\|_{L^{2}(a, b)}\right) \\
& \leq\left(\frac{1}{b-a}+2\right)\|\phi\|_{L^{2}(a, b)}\|\phi\|_{H^{1}(a, b)}
\end{aligned}
$$

This gives (4.5). If $\phi$ has a zero $x$, we apply (4.7) to obtain (4.6).

We are ready to derive the following a priori estimates for $\psi$. It can be understood as an analogue of Lemma 5 in [2].

LEMmA 4.3. Let $\psi$ be solution of (4.3) and $t \leq A$. Then

$$
t^{2}\left\|\psi^{\prime}\right\|_{L^{2}(P)}^{2}+A^{2}\left\|\psi-\omega^{\prime \prime}\right\|_{L^{2}(P)}^{2} \leq 6 A t\left\|\omega^{\prime \prime}\right\|_{H^{1}(P)}^{2} .
$$


Proof. Set $w:=\omega^{\prime \prime}$. Multiplying (4.3) by $-\psi^{\prime \prime}$ and integrating by parts, we obtain

$$
\begin{aligned}
t^{2}\left\|\psi^{\prime \prime}\right\|_{L^{2}(P)}^{2} & +A^{2}\left\|\psi^{\prime}\right\|_{L^{2}(P)} \\
& =A^{2} \int_{P} w^{\prime} \psi^{\prime} \mathrm{d} x-A^{2}\left[w(1 / 2) \psi^{\prime}(1 / 2)-w(-1 / 2) \psi^{\prime}(-1 / 2)\right] \\
& \leq A^{2}\left\|\psi^{\prime}\right\|_{L^{2}(P)}\left\|w^{\prime}\right\|_{L^{2}(P)}+2 A^{2}\left\|\psi^{\prime}\right\|_{L_{\infty}}\|w\|_{L_{\infty}} .
\end{aligned}
$$

By Rolle's theorem $\psi^{\prime}$ has a zero in $(-1 / 2,+1 / 2)$, and it follows from Lemma 4.2 that

$$
\left\|\psi^{\prime}\right\|_{L_{\infty}}^{2} \leq 2\left\|\psi^{\prime}\right\|_{L^{2}(P)}\left\|\psi^{\prime \prime}\right\|_{L^{2}(P)} .
$$

Young's inequality yields $\left\|\psi^{\prime}\right\|_{L^{2}(P)}\left\|w^{\prime}\right\|_{L^{2}(P)} \leq \frac{1}{4}\left\|\psi^{\prime}\right\|_{L^{2}(P)}^{2}+\left\|w^{\prime}\right\|_{L^{2}(P)}^{2}$ and with (4.10) also

$$
\begin{aligned}
A^{2}\left\|\psi^{\prime}\right\|_{L_{\infty}}\|w\|_{L_{\infty}} & \leq \frac{A t}{4}\left\|\psi^{\prime}\right\|_{L_{\infty}}^{2}+\frac{A^{3}}{t}\|w\|_{L_{\infty}}^{2} \\
& \leq \frac{1}{2} A t\left\|\psi^{\prime}\right\|_{L^{2}(P)}\left\|\psi^{\prime \prime}\right\|_{L^{2}(P)}+\frac{A^{3}}{t}\|w\|_{L_{\infty}}^{2} \\
& \leq \frac{1}{4}\left(t^{2}\left\|\psi^{\prime \prime}\right\|_{L^{2}(P)}^{2}+A^{2}\left\|\psi^{\prime}\right\|_{L^{2}(P)}^{2}\right)+\frac{A^{3}}{t}\|w\|_{L_{\infty}}^{2} .
\end{aligned}
$$

We insert the last two inequalities into (4.9) and note that the terms with the function $\psi$ can be absorbed by the left-hand side:

$$
\frac{1}{2} t^{2}\left\|\psi^{\prime \prime}\right\|_{L^{2}(P)}^{2}+\frac{1}{2} A^{2}\left\|\psi^{\prime}\right\|_{L^{2}(P)} \leq A^{2}\left\|w^{\prime}\right\|_{L^{2}(P)}^{2}+\frac{A^{3}}{t}\|w\|_{L_{\infty}}^{2} .
$$

Multiplying the above inequality by $t^{2} / A^{2}$ and using $(4.3)_{1}$ we obtain

$$
\frac{A^{2}}{2}\|\psi-w\|_{L^{2}(P)}^{2}+\frac{t^{2}}{2}\left\|\psi^{\prime}\right\|_{L^{2}(P)}^{2} \leq t^{2}\left\|w^{\prime \prime}\right\|_{L^{2}(P)}^{2}+A t\|w\|_{L_{\infty}}^{2} .
$$

Since $\left\|w^{\prime}\right\|_{L^{2}(P)} \leq\|w\|_{H^{1}(P)}$, the Gagliardo-Nirenberg inequality (4.5) yields the bound $\|w\|_{L_{\infty}} \leq \sqrt{3}\|w\|_{H^{1}(P)}$, and the right-hand side of (4.11) is smaller than $(1+\sqrt{3}) A t\|w\|_{H^{1}(P)}$. This completes the proof of (4.8).

As to (4.2), we have the a priori estimate

$$
\left\|\omega^{\prime \prime}\right\|_{H^{1}(P)} \leq C t^{-2}\|g\|_{H^{-1}(P)}, \quad\left\|\omega^{(4)}\right\|_{L^{2}(P)} \leq D^{-1} t^{-2}\|g\|_{L^{2}(P)} .
$$

THEOREM 4.4. Let $\hat{\boldsymbol{u}}$ be the beam mode defined in (4.1). There exists a constant $c=c[g]$ such that for sufficiently small $t$

$$
\frac{\|\boldsymbol{\epsilon}(\boldsymbol{u}-\hat{\boldsymbol{u}})\|_{\mathbb{C}}}{\|\boldsymbol{\epsilon}(\boldsymbol{u})\|_{\mathbb{C}}} \leq c t^{1 / 2} .
$$

Proof. For any $\boldsymbol{\tau} \in L^{2}\left(\Omega ; \mathbb{R}_{\text {sym }}^{2 \times 2}\right)$,

$$
\begin{aligned}
\langle\widehat{\boldsymbol{\sigma}}-\mathbb{C} \boldsymbol{\epsilon}(\hat{\boldsymbol{u}}), \boldsymbol{\tau}\rangle= & \left\langle\widehat{\sigma}_{11}-24 D \partial_{x} \hat{u}_{1}, \tau_{11}\right\rangle+\left\langle\widehat{\sigma}_{22}, \tau_{22}\right\rangle \\
& -\frac{\lambda}{\gamma}\left\langle\gamma \partial_{x} \hat{u}_{1}+\partial_{y} \hat{u}_{2}, \gamma \tau_{11}+\tau_{22}\right\rangle+2\left\langle\widehat{\sigma}_{12}-2 \mu \boldsymbol{\epsilon}_{12}(\hat{\boldsymbol{u}}), \tau_{12}\right\rangle .
\end{aligned}
$$


A direct calculation yields $\widehat{\sigma}_{11}-24 D \partial_{x} \hat{u}_{1}=0$. Therefore, we obtain

$$
\begin{aligned}
\langle\widehat{\boldsymbol{\sigma}}-\mathbb{C} \boldsymbol{\epsilon}(\hat{\boldsymbol{u}}), \boldsymbol{\tau}\rangle= & \left\langle\widehat{\sigma}_{22}, \tau_{22}\right\rangle-\frac{\lambda}{\gamma}\left\langle\gamma \partial_{x} \hat{u}_{1}+\partial_{y} \hat{u}_{2}, \gamma \tau_{11}+\tau_{22}\right\rangle \\
& +2\left\langle\widehat{\sigma}_{12}-2 \mu \boldsymbol{\epsilon}_{12}(\hat{\boldsymbol{u}}), \tau_{12}\right\rangle .
\end{aligned}
$$

By the definitions of $\widehat{\boldsymbol{\sigma}}$ and $\hat{\boldsymbol{u}}$ in (4.4) and (4.1), respectively, we have

$$
\begin{aligned}
& \left\|\widehat{\sigma}_{22}\right\|_{L^{2}\left(P_{t}\right)}^{2}=\frac{t^{3}}{3}\|g\|_{L^{2}(P)}^{2}, \quad\left\|\gamma \partial_{x} \hat{u}_{1}+\partial_{y} \hat{u}_{2}\right\|_{L^{2}(P)}^{2}=\frac{t^{3} \gamma^{2}}{12}\left\|\psi-\omega^{\prime \prime}\right\|_{L^{2}(P)}^{2}, \\
& \left\|\widehat{\sigma}_{12}-2 \mu \epsilon_{12}(\hat{\boldsymbol{u}})\right\|_{L^{2}\left(P_{t}\right)}^{2} \leq \frac{\mu^{2} \gamma^{2}}{60} t^{5}\left\|\psi^{\prime}\right\|_{L^{2}(P)}^{2}+2 \mu^{2}(1+\gamma)^{2} t^{5}\left\|\omega^{(3)}\right\|_{L^{2}(P)}^{2} .
\end{aligned}
$$

Substituting the above equations into (4.14) and using (4.8) and (4.12), we obtain

$$
\begin{aligned}
|\langle\widehat{\boldsymbol{\sigma}}-\mathbb{C} \boldsymbol{\epsilon}(\hat{\boldsymbol{u}}), \boldsymbol{\tau}\rangle| \leq C(\mu)\left[t^{3 / 2}\left(t\left\|\psi^{\prime}\right\|_{L^{2}(P)}+A\left\|\psi-\omega^{\prime \prime}\right\|_{L^{2}(P)}\right)\right. \\
\left.\quad+t^{5 / 2}\left\|\omega^{(3)}\right\|_{L^{2}(P)}+t^{3 / 2}\|g\|_{L^{2}(P)}\right]\|\boldsymbol{\tau}\|_{\mathbb{C}} \\
\leq C(\mu)\left(\|g\|_{H^{-1}(P)}+t^{3 / 2}\|g\|_{L^{2}(P)}\right)\|\boldsymbol{\tau}\|_{\mathbb{C}}
\end{aligned}
$$

It follows from Theorem 4.1 that

$$
\|\boldsymbol{\epsilon}(\boldsymbol{u}-\hat{\boldsymbol{u}})\|_{\mathbb{C}} \leq C(\mu)\left(\|g\|_{H^{-1}(P)}+t^{3 / 2}\|g\|_{L^{2}(P)}\right) .
$$

Recalling (2.6), we evaluate the denominator in (4.13),

$$
\begin{aligned}
\|\boldsymbol{\epsilon}(\hat{\boldsymbol{u}})\|_{\mathbb{C}}^{2} & =24 D\left\|y \omega^{\prime \prime}(x)\right\|_{L^{2}\left(P_{t}\right)}^{2}+\lambda \gamma\left\|y\left(\psi-\omega^{\prime \prime}\right)(x)\right\|_{L^{2}\left(P_{t}\right)}^{2}+\mu \gamma^{2}\left\|\phi(y) \psi^{\prime}(x)\right\|_{L^{2}\left(P_{t}\right)}^{2} \\
& =2 D t^{3}\left\|\omega^{\prime \prime}\right\|_{L^{2}(P)}^{2}+\frac{\lambda \gamma t^{3}}{12}\left\|\psi-\omega^{\prime \prime}\right\|_{L^{2}(P)}^{2}+\frac{\mu \gamma^{2} t^{5}}{320}\left\|\psi^{\prime}\right\|_{L^{2}(P)}^{2} .
\end{aligned}
$$

Using Lemma 4.3, we conclude that the second and the third term on the right-hand side of the above identity are bounded by a constant $C$ uniformly with respect to $t$. A simple scaling argument shows that

$$
\left\|\omega^{\prime \prime}\right\|_{L^{2}(P)}=c t^{-2},
$$

where the nonzero constant $c$ depends on $\|g\|_{H^{-1}(P)}$. Therefore,

$$
\|\boldsymbol{\epsilon}(\hat{\boldsymbol{u}})\|_{\mathbb{C}} \geq c t^{-1 / 2}
$$

holds with a nonzero constant $c$ that depends on $\|g\|_{H^{-1}(P)}$. From (4.16) and the triangle inequality it follows that

$$
\|\boldsymbol{\epsilon}(\boldsymbol{u})\|_{\mathbb{C}} \geq\|\boldsymbol{\epsilon}(\hat{\boldsymbol{u}})\|_{\mathbb{C}}-\|\boldsymbol{\epsilon}(\boldsymbol{u}-\hat{\boldsymbol{u}})\|_{\mathbb{C}} \geq \frac{1}{2} c t^{-1 / 2}
$$

holds for $t$ sufficiently small. By combining this inequality with (4.16) we complete the proof of (4.13).

We note that the constant in (4.13) depends on the quotient $\left\|\omega^{\prime \prime}\right\|_{H^{1}} /\left\|\omega^{\prime \prime}\right\|_{L_{2}}$. 
5. Discretization Error of the Beam Model. In this section we estimate the last two terms on the right-hand side of (3.15) and start with a construction of a finite element approximation $\boldsymbol{w} \in \boldsymbol{V}^{h}$. Let $\theta(x):=\omega^{\prime}(x), s(x):=1 / 4-x^{2}, \phi(y):=y^{2} / 2$, and

$$
\boldsymbol{w}=\left(w_{1}, w_{2}\right):=\left(-y \theta_{h}(x), \omega_{h}(x)+\gamma \phi_{h}(y) \psi_{h}(x)\right)
$$

where

$$
\begin{array}{r}
\theta_{h}:=\Pi_{1} \theta+\alpha \Pi_{1} s \quad \text { with } \quad \alpha:=\frac{\int_{P}\left(\theta-\Pi_{1} \theta\right)(x) \mathrm{d} x}{\int_{P} \Pi_{1} s(x) \mathrm{d} x}, \\
\phi_{h}:=\Pi_{1} \phi, \quad \psi_{h}:=\Pi \psi, \quad \omega_{h}(x):=\int_{-1 / 2}^{x} \Pi_{0} \theta_{h}\left(x^{\prime}\right) \mathrm{d} x^{\prime} .
\end{array}
$$

Here $\Pi_{1}$ is the standard linear interpolation operator and $\Pi$ the Clément interpolation operator [10].

By definition, $\omega_{h}(-1 / 2)=0$, and we have

$$
\begin{aligned}
\omega_{h}(1 / 2) & =\int_{P} \Pi_{0} \theta_{h}=\int_{P} \theta_{h}=\int_{P} \Pi_{1} \theta+\alpha \int_{P} \Pi_{1} s=\int_{P} \theta \\
& =\omega(1 / 2)-\omega(-1 / 2)=0 .
\end{aligned}
$$

By construction, we get $\theta_{h}( \pm 1 / 2)=0$. This shows that $\boldsymbol{w} \in \boldsymbol{V}^{h}$.

A standard finite element interpolation estimate yields

$$
\left\|\theta^{\prime}-\theta_{h}^{\prime}\right\|_{L^{2}(P)} \leq C h_{x}\left\|\theta^{\prime \prime}\right\|_{L^{2}(P)}=C h_{x}\left\|\omega^{(3)}\right\|_{L^{2}(P)} .
$$

From $\phi(y) \leq t^{2} / 8$ it follows that also $\phi_{h}(y) \leq t^{2} / 8$ and

$$
\left\|\phi_{h}\right\|_{L^{2}\left(I_{t}\right)} \leq \frac{1}{8} t^{5 / 2}
$$

The following lemma is the key to an estimate of the interpolation error.

Lemma 5.1. Let $\psi_{h}$ be defined in (5.1). There holds

$$
\begin{aligned}
\left\|\psi-\psi_{h}\right\|_{L^{2}(P)} & \leq C\left(\left\|\psi-\omega^{\prime \prime}\right\|_{L^{2}(P)}+h_{x}\left\|\omega^{(3)}\right\|_{L^{2}(P)}\right), \\
\left\|\psi-\Pi_{x} \psi\right\|_{L^{2}(P)} & \leq\left\|\psi-\omega^{\prime \prime}\right\|_{L^{2}(P)}+C h_{x}\left\|\omega^{(3)}\right\|_{L^{2}(P)} .
\end{aligned}
$$

Proof. We write

$$
\psi-\psi_{h}=(I-\Pi)\left(\psi-\omega^{\prime \prime}\right)+(I-\Pi) \omega^{\prime \prime} .
$$

By the following properties of the Clément operator [10],

$$
\|\Pi \psi\|_{L^{2}(P)} \leq C\|\psi\|_{L^{2}(P)}, \quad\left\|(I-\Pi) \omega^{\prime \prime}\right\|_{L^{2}(P)} \leq C h_{x}\left\|\omega^{(3)}\right\|_{L^{2}(P)},
$$

we get the first estimate (5.4).

Proceeding along the same line and using the above estimate with $\Pi$ replaced by $\Pi_{x}$, we obtain $(5.5)$. $\square$

It is our main task to show that locking phenomena are eliminated. Note that the shear term of the beam model

$$
\boldsymbol{\epsilon}_{12}(\hat{\boldsymbol{u}})=\frac{\gamma}{2} y^{2} \psi^{\prime}(x)
$$


is small. It is crucial that the reduced shear term of the finite element interpolant $\Pi_{0} \boldsymbol{\epsilon}_{12}(\boldsymbol{w})$ is also small.

Lemma 5.2. Let $\boldsymbol{w}$ be defined by (5.1). Then

$$
\|\hat{\boldsymbol{u}}-\boldsymbol{w}\| \leq C\left(1+h_{x} / t^{1 / 2}\right)\|g\|_{H^{-1}(P)} .
$$

Proof. It follows from

$$
\phi_{h}^{\prime}(y)=f_{I_{y}} \phi_{h}^{\prime}(\tilde{y}) d \tilde{y}=f_{I_{y}} \phi^{\prime}(\tilde{y}) d \tilde{y}=\Pi_{y} \phi^{\prime}
$$

that $\Pi_{0}\left[\phi_{h}^{\prime}(y) \psi_{h}(x)\right]=\Pi_{0}\left[\phi^{\prime}(y) \psi_{h}(x)\right]$ and

$$
\begin{aligned}
\| \Pi_{0}\left[\gamma \partial_{x}\left(\hat{u}_{1}-w_{1}\right)\right. & \left.+\partial_{y}\left(\hat{u}_{2}-w_{2}\right)\right] \|_{L^{2}\left(P_{t}\right)} \\
& \left.\leq \gamma\left\|\partial_{x}\left(\hat{u}_{1}-w_{1}\right)\right\|_{L^{2}\left(P_{t}\right)}+\gamma \| \phi^{\prime}(y) \Pi_{x}\left(\psi-\psi_{h}\right)(x)\right] \|_{L^{2}\left(P_{t}\right)} .
\end{aligned}
$$

Invoking (5.7) once more, we get

$$
\left(I-\Pi_{x}\right) \partial_{y}\left(\hat{u}_{2}-w_{2}\right)=\left(I-\Pi_{y}\right) \phi^{\prime}\left(I-\Pi_{x}\right) \psi+\Pi_{y} \phi^{\prime}\left(I-\Pi_{x}\right)\left(\psi-\psi_{h}\right) .
$$

This leads to

$$
\left\|\left(I-\Pi_{x}\right) \partial_{y}\left(\hat{u}_{2}-w_{2}\right)\right\|_{L^{2}\left(P_{t}\right)} \leq\left\|\phi^{\prime}\left(I-\Pi_{x}\right) \psi\right\|_{L^{2}\left(P_{t}\right)}+\left\|\phi^{\prime}\left(\psi-\psi_{h}\right)\right\|_{L^{2}\left(P_{t}\right)} .
$$

Obviously,

$$
\left\|\Pi_{0}\left[\partial_{y}\left(\hat{u}_{1}-w_{1}\right)+\partial_{x}\left(\hat{u}_{2}-w_{2}\right)\right]\right\|_{L^{2}\left(P_{t}\right)} \leq\left\|\boldsymbol{\epsilon}_{12}(\hat{\boldsymbol{u}})\right\|_{L^{2}\left(P_{t}\right)}+\left\|\Pi_{0} \boldsymbol{\epsilon}_{12}(\boldsymbol{w})\right\|_{L^{2}\left(P_{t}\right)} .
$$

Combining the above three inequalities with (3.2) and (2.3) we have

$$
\begin{aligned}
\|\hat{\boldsymbol{u}}-\boldsymbol{w}\| \leq 2 \sqrt{2 \lambda / \gamma}[ & \left\|\partial_{x}\left(\hat{u}_{1}-w_{1}\right)\right\|_{L^{2}\left(P_{t}\right)}+\left\|\boldsymbol{\epsilon}_{12}(\hat{\boldsymbol{u}})\right\|_{L^{2}\left(P_{t}\right)}+\left\|\Pi_{0} \boldsymbol{\epsilon}_{12}(\boldsymbol{w})\right\|_{L^{2}\left(P_{t}\right)} \\
& \left.+\left\|\phi^{\prime}\left(\psi-\psi_{h}\right)\right\|_{L^{2}\left(P_{t}\right)}+\left\|\phi^{\prime}\left(I-\Pi_{x}\right) \psi\right\|_{L^{2}\left(P_{t}\right)}\right] .
\end{aligned}
$$

By (5.2),

$$
\left\|\partial_{x}\left(\hat{u}_{1}-w_{1}\right)\right\|_{L^{2}\left(P_{t}\right)}=\left\|y\left(\theta^{\prime}-\theta_{h}^{\prime}\right)\right\|_{L^{2}\left(P_{t}\right)} \leq C h_{x} t^{3 / 2}\left\|\omega^{(3)}\right\|_{L^{2}(P)} .
$$

A direct calculation leads to

$$
\left\|\boldsymbol{\epsilon}_{12}(\hat{\boldsymbol{u}})\right\|_{L^{2}\left(P_{t}\right)}=\frac{\gamma}{2}\|\phi\|_{L^{2}\left(I_{t}\right)}\left\|\psi^{\prime}\right\|_{L^{2}(P)} \leq C t^{5 / 2}\left\|\psi^{\prime}\right\|_{L^{2}(P)},
$$

and

$$
\Pi_{0} \boldsymbol{\epsilon}_{12}(\boldsymbol{w})=\frac{\gamma}{2} \Pi_{0}\left[\phi_{h}(y) \psi_{h}^{\prime}(x)\right]+\frac{1}{2} \Pi_{0}\left(\theta_{h}-\Pi_{0} \theta_{h}\right)=\frac{\gamma}{2} \Pi_{0}\left[\phi_{h}(y) \psi_{h}^{\prime}(x)\right] .
$$

It follows from (5.3) and $\left\|\psi_{h}^{\prime}\right\|_{L^{2}(P)} \leq C\left\|\psi^{\prime}\right\|_{L^{2}(P)}$ that

$$
\left\|\Pi_{0} \boldsymbol{\epsilon}_{12}(\boldsymbol{w})\right\|_{L^{2}\left(P_{t}\right)} \leq \frac{\gamma}{2}\left\|\phi_{h}(y) \psi_{h}^{\prime}(x)\right\|_{L^{2}\left(P_{t}\right)} \leq C t^{5 / 2}\left\|\psi^{\prime}\right\|_{L^{2}(P)} .
$$

By (5.4) and (5.5), the last two terms in (5.8) are bounded by

$$
\left\|\phi^{\prime}\left(\psi-\psi_{h}\right)\right\|_{L^{2}\left(P_{t}\right)}+\left\|\phi^{\prime}\left(I-\Pi_{x}\right) \psi\right\|_{L^{2}\left(P_{t}\right)} \leq C t^{3 / 2}\left(\left\|\psi-\omega^{\prime \prime}\right\|_{L^{2}(P)}+h_{x}\left\|\omega^{(3)}\right\|_{L^{2}(P)}\right) \text {. }
$$


Summing up the above estimates for the terms on the right-hand side of (5.8), and using the a priori estimate (4.8), we obtain

$$
\begin{aligned}
\|\hat{\boldsymbol{u}}-\boldsymbol{w}\| & \leq C t^{3 / 2}\left(\left\|\psi-\omega^{\prime \prime}\right\|_{L^{2}(P)}+t\left\|\psi^{\prime}\right\|_{L^{2}(P)}+h_{x}\left\|\omega^{(3)}\right\|_{L^{2}(P)}\right) \\
& \leq C\left(1+h_{x} / t^{1 / 2}\right)\|g\|_{H^{-1}(P)} .
\end{aligned}
$$

In Theorem 4.4, we have shown that the quantity

$$
\frac{\left\langle\mathbb{C}^{-1}(\widehat{\boldsymbol{\sigma}}-\mathbb{C} \boldsymbol{\epsilon}(\hat{\boldsymbol{u}})), \widehat{\boldsymbol{\sigma}}-\mathbb{C} \boldsymbol{\epsilon}(\hat{\boldsymbol{u}})\right\rangle^{1 / 2}}{\|\boldsymbol{\epsilon}(\boldsymbol{u})\|_{\mathbb{C}}}
$$

is small. Next we show that it remains small with $\mathbb{C}$ replaced by $\mathbb{C}_{h}$.

Lemma 5.3. Let $\widehat{\boldsymbol{\sigma}}$ and $\hat{\boldsymbol{u}}$ be defined as in (4.4) and (4.1), respectively. Then

$$
\sup _{\boldsymbol{w} \in \boldsymbol{V}^{h}} \frac{\left|\left\langle\widehat{\boldsymbol{\sigma}}-\mathbb{C}_{h} \boldsymbol{\epsilon}(\hat{\boldsymbol{u}}), \boldsymbol{\epsilon}(\boldsymbol{w})\right\rangle\right|}{\|\boldsymbol{w}\|} \leq C\left(\left(1+h_{x} / t^{1 / 2}\right)\|g\|_{H^{-1}(P)}+t^{3 / 2}\|g\|_{L^{2}(P)}\right) .
$$

Proof. Similar to (4.14), we have the following expansion

$$
\begin{aligned}
\langle\widehat{\boldsymbol{\sigma}}- & \left.\mathbb{C}_{h} \boldsymbol{\epsilon}(\hat{\boldsymbol{u}}), \boldsymbol{\epsilon}(\boldsymbol{w})\right\rangle=\left\langle\widehat{\sigma}_{22}-24 D\left(I-\Pi_{x}\right) \partial_{y} \hat{u}_{2}, \partial_{y} w_{2}\right\rangle \\
& -\frac{\lambda}{\gamma}\left\langle\Pi_{0}\left[\gamma \partial_{x} \hat{u}_{1}+\partial_{y} \hat{u}_{2}\right], \gamma \partial_{x} w_{1}+\partial_{y} w_{2}\right\rangle+2\left\langle\widehat{\sigma}_{12}-2 \mu \Pi_{0} \boldsymbol{\epsilon}_{12}(\hat{\boldsymbol{u}}), \boldsymbol{\epsilon}_{12}(\boldsymbol{w})\right\rangle,
\end{aligned}
$$

which may be rewritten as

$$
\begin{aligned}
\langle\widehat{\boldsymbol{\sigma}}- & \left.\mathbb{C}_{h} \boldsymbol{\epsilon}(\hat{\boldsymbol{u}}), \boldsymbol{\epsilon}(\boldsymbol{w})\right\rangle=\left\langle\widehat{\sigma}_{22}, \Pi_{0} \partial_{y} w_{2}\right\rangle-\frac{\lambda}{\gamma}\left\langle\gamma \partial_{x} \hat{u}_{1}+\partial_{y} \hat{u}_{2}, \Pi_{0}\left[\gamma \partial_{x} w_{1}+\partial_{y} w_{2}\right]\right\rangle \\
& +2\left\langle\widehat{\sigma}_{12}-2 \mu \boldsymbol{\epsilon}_{12}(\hat{\boldsymbol{u}}), \Pi_{0} \boldsymbol{\epsilon}_{12}(\boldsymbol{w})\right\rangle+2\left\langle\left(I-\Pi_{0}\right) \widehat{\sigma}_{12}, \boldsymbol{\epsilon}_{12}(\boldsymbol{w})\right\rangle \\
& +\left\langle\widehat{\sigma}_{22},\left(I-\Pi_{0}\right) \partial_{y} w_{2}\right\rangle-24 D\left\langle\left(I-\Pi_{x}\right) \partial_{y} \hat{u}_{2}, \partial_{y} w_{2}\right\rangle \\
= & {\left[\left\langle\widehat{\boldsymbol{\sigma}}-\mathbb{C} \boldsymbol{\epsilon}(\hat{\boldsymbol{u}}), \Pi_{0} \boldsymbol{\epsilon}(\boldsymbol{w})\right\rangle+\left\langle\widehat{\sigma}_{22},\left(I-\Pi_{x}\right) \partial_{y} w_{2}\right\rangle\right] } \\
& +2\left\langle\widehat{\sigma}_{12},\left(I-\Pi_{0}\right) \boldsymbol{\epsilon}_{12}(\boldsymbol{w})\right\rangle-24 D\left\langle\left(I-\Pi_{x}\right) \partial_{y} \hat{u}_{2}, \partial_{y} w_{2}\right\rangle .
\end{aligned}
$$

It is clear to see

$$
2\left\langle\widehat{\sigma}_{12},\left(I-\Pi_{0}\right) \boldsymbol{\epsilon}_{12}(\boldsymbol{w})\right\rangle=\left\langle\widehat{\sigma}_{12},\left(I-\Pi_{x}\right) \partial_{y} w_{1}\right\rangle+\left\langle\widehat{\sigma}_{12},\left(I-\Pi_{0}\right) \partial_{x} w_{2}\right\rangle .
$$

We use $\left(I-\Pi_{x}\right) \partial_{y} w_{1}=\partial_{y}\left(I-\Pi_{x}\right) w_{1}$, integrate by parts, note that $\left(I-\Pi_{x}\right) w_{1}$ is continuous in $y$ and $\widehat{\sigma}_{12}$ vanishes on $\partial P_{t}^{ \pm}$, to obtain

$$
\begin{aligned}
\left\langle\widehat{\sigma}_{12}, \partial_{y}\left(I-\Pi_{x}\right) w_{1}\right\rangle & =-\left\langle\partial_{y} \widehat{\sigma}_{12},\left(I-\Pi_{x}\right) w_{1}\right\rangle+\int_{\partial P_{t}^{ \pm}} \widehat{\sigma}_{12} n_{y}\left(I-\Pi_{x}\right) w_{1} \mathrm{~d} x \\
& =-\left\langle\partial_{y} \widehat{\sigma}_{12},\left(I-\Pi_{x}\right) w_{1}\right\rangle .
\end{aligned}
$$

Here $\boldsymbol{n}=\left(n_{x}, n_{y}\right)$ is the outward unit normal to $\partial P_{t}^{ \pm}$. We proceed applying the Cauchy-Schwarz inequality

$$
\begin{aligned}
\left|\left\langle\widehat{\sigma}_{12}, \partial_{y}\left(I-\Pi_{x}\right) w_{1}\right\rangle\right| & \leq\left\|\partial_{y} \widehat{\sigma}_{12}\right\|_{L^{2}\left(P_{t}\right)}\left\|\left(I-\Pi_{x}\right) w_{1}\right\|_{L^{2}\left(P_{t}\right)} \\
& \leq C(24 D) h_{x} t^{3 / 2}\left\|\omega^{(3)}\right\|_{L^{2}(P)}\left\|\partial_{x} w_{1}\right\|_{L^{2}\left(P_{t}\right)} \\
& \leq C h_{x} t^{-1 / 2}\|g\|_{H^{-1}(P)}\|\boldsymbol{w}\| .
\end{aligned}
$$


Moreover, it follows from (4.12) that $\left\|\widehat{\sigma}_{12}\right\|_{L^{2}\left(P_{t}\right)} \leq C t^{1 / 2}\|g\|_{H^{-1}(P)}$, which together with $(3.12)_{2}$ leads to

$$
\begin{aligned}
\left|\left\langle\widehat{\sigma}_{12},\left(I-\Pi_{0}\right) \partial_{x} w_{2}\right\rangle\right| & \leq\left\|\widehat{\sigma}_{12}\right\|_{L^{2}\left(P_{t}\right)}\left\|\left(I-\Pi_{0}\right) \partial_{x} w_{2}\right\|_{L^{2}\left(P_{t}\right)} \\
& \leq C t^{1 / 2}\|g\|_{H^{-1}(P)}\left\|\left(I-\Pi_{x}\right) \partial_{y} w_{2}\right\|_{L^{2}\left(P_{t}\right)} \\
& \leq C t^{1 / 2}\|g\|_{H^{-1}(P)}\|\boldsymbol{w}\| .
\end{aligned}
$$

Combining the above two estimates, we obtain

$$
\left|2\left\langle\widehat{\sigma}_{12},\left(I-\Pi_{0}\right) \boldsymbol{\epsilon}_{12}(\boldsymbol{w})\right\rangle\right| \leq C\left(h_{x} / t^{1 / 2}+t^{1 / 2}\right)\|g\|_{H^{-1}(P)}|\|\boldsymbol{w} \mid\| .
$$

It follows from (5.5) that

$$
\begin{aligned}
\left|\left\langle\left(I-\Pi_{x}\right) \partial_{y} \hat{u}_{2}, \partial_{y} w_{2}\right\rangle\right| & \leq C t^{3 / 2}\left\|\psi-\Pi_{x} \psi\right\|_{L^{2}(P)}\left\|\partial_{y} w_{2}\right\|_{L^{2}\left(P_{t}\right)} \\
& \leq C t^{3 / 2}\left(\left\|\psi-\omega^{\prime \prime}\right\|_{L^{2}(P)}+C h_{x}\left\|\omega^{(3)}\right\|_{L^{2}(P)}\right)\|\boldsymbol{w}\| \\
& \leq C\left(1+h_{x} / t^{1 / 2}\right)\|g\|_{H^{-1}(P)}\|\boldsymbol{w}\| .
\end{aligned}
$$

Using $(3.12)_{1},(4.15)$ and $\left\|\Pi_{0} \boldsymbol{\epsilon}(\boldsymbol{w})\right\|_{\mathbb{C}} \leq\|\boldsymbol{w}\|$, we bound the terms in the square bracket of (5.10) as

$$
\begin{aligned}
& \left|\left\langle\widehat{\boldsymbol{\sigma}}-\mathbb{C} \boldsymbol{\epsilon}(\hat{\boldsymbol{u}}), \Pi_{0} \boldsymbol{\epsilon}(\boldsymbol{w})\right\rangle+\left\langle\widehat{\sigma}_{22},\left(I-\Pi_{x}\right) \partial_{y} w_{2}\right\rangle\right| \\
\leq & C t^{3 / 2}\|g\|_{L^{2}(P)}\left\|\left(I-\Pi_{x}\right) \partial_{y} w_{2}\right\|_{L^{2}\left(P_{t}\right)}+C\left(\|g\|_{H^{-1}(P)}+t^{3 / 2}\|g\|_{L^{2}(P)}\right)\|\boldsymbol{w}\| \\
\leq & C\left(\|g\|_{H^{-1}(P)}+t^{3 / 2}\|g\|_{L^{2}(P)}\right)\|\boldsymbol{w}\| .
\end{aligned}
$$

By inserting the above estimate, (5.12), and (5.11) in (5.10) we complete the proof of (5.9).

Now we are ready to establish the main result.

THEOREM 5.4. There exists $c=c[g]$ independent of $t$ and $\rho$ such that for sufficiently small $t$

$$
\frac{\left\|\boldsymbol{u}-\boldsymbol{u}_{h}\right\|}{\|\boldsymbol{\epsilon}(\boldsymbol{u})\|_{\mathbb{C}}} \leq c\left(t^{1 / 2}+h_{x}\right)
$$

Proof. The substitution of (5.6) and (5.9) into (3.15) leads to

$$
\left\|\boldsymbol{u}-\boldsymbol{u}_{h}\right\| \leq C\left(1+h_{x} / t^{1 / 2}\right)\|g\|_{H^{-1}(P)}+C t^{3 / 2}\|g\|_{L^{2}(P)}+\|\boldsymbol{\epsilon}(\boldsymbol{u}-\hat{\boldsymbol{u}})\|_{\mathbb{C}} .
$$

Using (4.13) to estimate the last term on the right-hand side of the above inequality and recalling (4.18), we get (5.13).

Proceeding along the same lines that led to (5.13), using (3.14), (4.17), and (4.16), we get

$$
\begin{aligned}
\|\boldsymbol{u}\| & \geq\|\hat{\boldsymbol{u}}\||-\|\boldsymbol{u}-\hat{\boldsymbol{u}}\|| \\
& \geq \sqrt{24 D}\left\|\partial_{x} \hat{u}_{1}\right\|_{L^{2}\left(P_{t}\right)}-\sqrt{1+\gamma}\|\boldsymbol{\epsilon}(\boldsymbol{u}-\hat{\boldsymbol{u}})\|_{\mathbb{C}} \\
& \geq c t^{-1 / 2}
\end{aligned}
$$

for a nonzero constant $c$ that depends on $\|g\|_{H^{-1}(P)}$. This immediately implies 
Corollary 5.5. There exists $c=c[g]$ independent of $t$ and $\rho$ such that for sufficiently small $t$

$$
\frac{\left\|\boldsymbol{u}-\boldsymbol{u}_{h}\right\| \mid}{\|\boldsymbol{u}\| \mid} \leq c\left(t^{1 / 2}+h_{x}\right)
$$

In view of the equivalence between the enhanced strain finite element and the method of incompatible modes [24] over the rectangular mesh, we have actually proved that the Wilson nonconforming elements $[28,25]$ are also free of shear locking in the thin beam limit.

We have restricted ourselves to rectangular grids. This is in accordance with the observation that the enhanced assumed strain method of Simo AND RIFAI [24] may suffer from trapezoidal locking on general quadrilateral meshes.

6. Conclusion and Perspective. In this paper, we have investigated the bending dominated plane elasticity problem and proved that the enhanced assumed strain method (EAS method) yields finite element solutions with an order of approximation that is at least as good as a beam model with dimension reduction. The convergence result holds also for meshes with high element aspect ratio, which is common when solving problems on thin domains. In this way we exclude shear locking although a generic constant in the error estimate depends on the smoothness of the load. The main result, Theorem 5.4 may also apply to other enhanced assumed strain methods as discussed in [5].

Appendix A. A Negative Result for the Triple Norm. The error bound in our main result heavily depends on the right choice of the reduced energy. If we replace $\Pi_{x}$ in the last term of $\||\cdot| \mid$ by $\Pi_{0}$, and denote the modified norm by $\|\cdot\|_{*}$, then we have the following lower bound for the interpolation error in this norm, which plays the same role as the lower bound in [6, Theorem 3] for the volumetric locking.

Lemma A.1. Let $\hat{\boldsymbol{u}}$ be the beam model defined in (4.1). Then

$$
\inf _{\boldsymbol{w} \in \boldsymbol{V}^{h}}\|\hat{\boldsymbol{u}}-\boldsymbol{w}\| \|_{*} \geq C h_{y} t^{-3 / 2} .
$$

Proof. For any $\boldsymbol{w} \in \boldsymbol{V}^{h}$, we have

$$
\|\hat{\boldsymbol{u}}-\boldsymbol{w}\|_{*}^{2} \geq\|\hat{\boldsymbol{u}}-\boldsymbol{w}\|_{*}^{2}-\|\hat{\boldsymbol{u}}-\boldsymbol{w}\|^{2}=24 D\left\|\left(\Pi_{x}-\Pi_{0}\right) \partial_{y}\left(\hat{u}_{2}-w_{2}\right)\right\|_{L^{2}\left(P_{t}\right)}^{2} .
$$

Noting that $\left(\Pi_{x}-\Pi_{0}\right) \partial_{y} w_{2}=0$ we get

$$
\inf _{\boldsymbol{w} \in \boldsymbol{V}^{h}}\|\hat{\boldsymbol{u}}-\boldsymbol{w}\|\left\|_{*} \geq \sqrt{24 D}\right\|\left(\Pi_{x}-\Pi_{0}\right) \partial_{y} \hat{u}_{2} \|_{L^{2}\left(P_{t}\right)} .
$$

A straightforward calculation gives

$$
\begin{aligned}
24 D\left\|\left(\Pi_{x}-\Pi_{0}\right) \partial_{y} \hat{u}_{2}\right\|_{L^{2}\left(P_{t}\right)}^{2} & =24 D\left\|\left(I-\Pi_{y}\right) \phi^{\prime}\right\|_{L^{2}\left(I_{t}\right)}^{2}\left\|\Pi_{x} \psi\right\|_{L^{2}(P)}^{2} \\
& =2 D h_{y}^{2} t\left\|\Pi_{x} \psi\right\|_{L^{2}(P)}^{2} .
\end{aligned}
$$

Proceeding along the same line as that leading to (5.5) and using the a priori estimate (4.8) and (4.17), we obtain for sufficiently small $t$ and $h_{x}$

$$
\begin{aligned}
\left\|\Pi_{x} \psi\right\|_{L^{2}(P)} & \geq\left\|\omega^{\prime \prime}\right\|_{L^{2}(P)}-\left\|\left(I-\Pi_{x}\right) \omega^{\prime \prime}\right\|_{L^{2}(P)}-\left\|\Pi_{x}\left(\psi-\omega^{\prime \prime}\right)\right\|_{L^{2}(P)} \\
& \geq c t^{-2}-c h_{x}\left\|\omega^{(3)}\right\|_{L^{2}(P)}-\left\|\psi-\omega^{\prime \prime}\right\|_{L^{2}(P)} \\
& \geq c t^{-2}\left(1-h_{x}-t^{1 / 2}\right) \\
& \geq(c / 2) t^{-2} .
\end{aligned}
$$


The substitution of the above two inequalities in (A.2) yields (A.1). $\mathrm{u}$

Based on the above lemma, we shall obtain a lower bound for the relative error in the norm $\|\mid \cdot\|_{*}$. For any $\boldsymbol{w}, \boldsymbol{v} \in \boldsymbol{V}$, let

$$
\left\langle\tilde{\mathbb{C}}_{h} \boldsymbol{\epsilon}(\boldsymbol{w}), \boldsymbol{\epsilon}(\boldsymbol{w})\right\rangle:=\|\boldsymbol{w}\|_{*}^{2},
$$

and $\left\langle\tilde{\mathbb{C}}_{h} \boldsymbol{\epsilon}(\boldsymbol{w}), \boldsymbol{\epsilon}(\boldsymbol{v})\right\rangle$ be the associated bilinear form. Proceeding along the same way as for the derivation of (3.14), we obtain

$$
\|\boldsymbol{v}\|_{*} \leq \sqrt{1+\gamma}\|\boldsymbol{\epsilon}(\boldsymbol{v})\|_{\mathbb{C}} \quad \text { for any } \quad \boldsymbol{v} \in \boldsymbol{V} .
$$

Lemma A.2. There exists a constant c that depends on $\|g\|_{H^{-1}(P)}$ but independent of $t, \rho$ and $h$ such that

$$
\frac{\left\|\boldsymbol{u}-\boldsymbol{u}_{h}\right\|_{*}}{\|\boldsymbol{\epsilon}(\boldsymbol{u})\|_{\mathbb{C}}} \geq c
$$

Proof. Let $\tilde{\boldsymbol{u}} \in \boldsymbol{V}^{h}$ be the solution of

$$
\left\langle\tilde{\mathbb{C}}_{h} \boldsymbol{\epsilon}(\tilde{\boldsymbol{u}}), \boldsymbol{\epsilon}(\boldsymbol{w})\right\rangle=\left\langle\tilde{\mathbb{C}}_{h} \boldsymbol{\epsilon}(\hat{\boldsymbol{u}}), \boldsymbol{\epsilon}(\boldsymbol{w})\right\rangle \quad \text { for all } \boldsymbol{w} \in \boldsymbol{V}^{h} .
$$

By the Galerkin orthogonality, we obtain

$$
\left\|\hat{\boldsymbol{u}}-\boldsymbol{u}_{h}\right\|_{*}^{2}=\|\hat{\boldsymbol{u}}-\tilde{\boldsymbol{u}}\|_{*}^{2}+\left\|\tilde{\boldsymbol{u}}-\boldsymbol{u}_{h}\right\|_{*}^{2}=\inf _{\boldsymbol{w} \in V^{h}}\|\hat{\boldsymbol{u}}-\boldsymbol{w}\|_{*}^{2}+\left\|\tilde{\boldsymbol{u}}-\boldsymbol{u}_{h}\right\|_{*}^{2},
$$

which immediately implies

$$
\left\|\hat{\boldsymbol{u}}-\boldsymbol{u}_{h}\right\|_{*} \geq \inf _{\boldsymbol{w} \in \boldsymbol{V}^{h}}\|\hat{\boldsymbol{u}}-\boldsymbol{w}\|_{*} .
$$

It implies together with the triangle inequality, the inequality (A.3), Lemma A.1, and Theorem 4.4 for sufficiently small $t$ :

$$
\begin{aligned}
\left\|\boldsymbol{u}-\boldsymbol{u}_{h}\right\|_{*} & \geq\left\|\hat{\boldsymbol{u}}-\boldsymbol{u}_{h} \mid\right\|_{*}-\|\boldsymbol{u}-\hat{\boldsymbol{u}}\|_{*} \\
& \geq \inf _{\boldsymbol{w} \in \boldsymbol{V}^{h}}\|\hat{\boldsymbol{u}}-\boldsymbol{w}\|_{*}-\sqrt{1+\gamma}\|\boldsymbol{\epsilon}(\boldsymbol{u}-\hat{\boldsymbol{u}})\|_{\mathbb{C}} \\
& \geq c h_{y} / t^{3 / 2} .
\end{aligned}
$$

Recalling (4.18) we get (A.4).

Remark A.3. In view of Lemma A.1 and Lemma 5.2, we have

$$
\inf _{\boldsymbol{w} \in \boldsymbol{V}^{h}} \frac{\|\hat{\boldsymbol{u}}-\boldsymbol{w}\|}{\|\boldsymbol{\epsilon}(\boldsymbol{u})\|_{\mathbb{C}}} \leq C\left(t^{1 / 2}+h_{x}\right)<C \frac{h_{y}}{t} \leq \inf _{\boldsymbol{w} \in \boldsymbol{V}^{h}} \frac{\|\hat{\boldsymbol{u}}-\boldsymbol{w}\|_{*}}{\|\boldsymbol{\epsilon}(\boldsymbol{u})\|_{\mathbb{C}}},
$$

which shows that the alternate triple norm $\|\mid \cdot\|_{*}$ dos not soften the strain energy sufficiently enough such that the approximation result holds, while the triple norm $\||\cdot| \mid$, indeed, reduces the strain energy to the desired degree.

\section{REFERENCES}

[1] R.A. Adams and J.J.F. Fournier, Sobolev Spaces, 2nd ed., Academic Press, 2003. 
[2] A.L. Alessandrini, D.N. Arnold, R.S. Falk, and A.L. Madureira, Derivation and justification of plate models by variational methods, In "Plates and Shells, Quebec 1996", (M. Fortin, ed.), pp. 1-20. CRM Proceeding and Lecture Notes, vol. 21, American Mathematical Society, Providence, RI. (1999)

[3] T. Belytschko and W.E. Bachrach, Efficient implementation of quadrilaterals with high coarsemesh accuracy, Comput. Methods Appl. Mech. Engrg. 54(1986), 279-301.

[4] A. Berger, R. Scott and G. Strang, Approximate boundary conditions in the finite element method, Symposia Mathematica X(1972), 295-313.

[5] M. Bischoff, E. Ramm and D. Braess, A class of equivalent enhanced assumed strain and hybrid stress finite elements, Comput. Mech. 22(1999), 443-449.

[6] D. Braess, Enhanced assumed strain elements and locking in membrane problems, Comput. Methods Appl. Mech. Engrg. 165 (1998), 155-174.

[7] D. Braess, Finite Elements, Theory, Fast Solvers, and Applications in Solid Mechanics, Cambridge University Press, Cambridge, 3rd ed., 2007.

[8] D. Braess, C. Carstensen and B.D. Reddy, Uniform convergence and a posteriori error estimators for the enhanced strain finite element method, Numer. Math. 96 (2004), 461-479.

[9] P.G. Ciarlet, Mathematical Elasticity II: Theory of Plates, Elsevier Science B.V., 1997.

[10] P. Clément, Approximation by finite element functions using local regularization, Rev. Française Automat. Infomat. Recherche Opérationnelle Sér. Rouge Anal. Numér. 9(R-2) (1975), 77-84.

[11] P. Destuynder, Estimations d'erreur explicites pour les modèles de plaques de Kirchhoff-Love et Reissner-Mindlin, C.R. Acad. Sci. Paris, t.325, Série I, (1997), 233-238.

[12] B.P. Lamichhane, B.D. Reddy and B.I. Wohlmuth, Convergence in the incompressible limit of finite element approximations based on the Hu-Washizu formulation, Numer. Math. 104 (2006), 151-175.

[13] C. Lovadina, Analysis of strain-pressure finite element methods for the Stokes problem, Numer. Methods Partial Diff. Equations 13 (1997), 717-730.

[14] R.H. MacNeal, A theorem regarding the locking of tapered four-noded membrane elements, Internat. J. Numer. Methods Engrg. 24 (1987), 1793-1799.

[15] R.H. MacNeal, Finite Elements: Their Design and Performance, Marcel Dekker, New York, 1994.

[16] J.E. Marsden and T.J.R. Hughes, Mathematical Foundations of Elasticity, Dover Publications Inc., New York, 1994.

[17] D. Morgenstern, Herleitung der Plattentheorie aus der dreidimensionalen Elastizitatstheorie, Arch. Ration. Mech. Anal. 4 (1959), 145-152.

[18] J. Pitkäranta, A first locking-free plane-elastic finite element: historia mathematica, Comput. Methods Appl. Mech. Engrg. 190 (2000), 1323-1366.

[19] W. Prager and J.L. Synge, Approximations in elasticity based on the concept of function space, Quart. Appl. Math. 5 (1947), 241-269.

[20] B.D. Reddy and J.C. Simo, Stability and convergence of a class of enhanced strain methods, SIAM J. Numer. Anal. 32 (1995), 1705-1728.

[21] Ch. Schwab: Hierarchical Models of Plates - Fourier Analysis and A-posteriori error estimation. Habilitation Thesis, Stuttgart, Germany (1995).

[22] J.C. Simo and F. Armero, Geometrically nonlinear enhanced strain mixed methods and the method of incompatible modes, Internat. J. Numer. Methods Engrg. 33 (1992), 1413-1449.

[23] J.C. Simo, F. Armero and R.L. Taylor, Improved versions of assumed enhanced strain trilinear elements for $3 D$ finite deformation problems, Comput. Methods Appl. Mech. Engrg. 110 (1993), 359-386.

[24] J.C. Simo and M.S. Rifai, A class of mixed assumed strain methods and the method of incompatible modes, Internat. J. Numer. Methods Engrg. 29 (1990), 1595-1638.

[25] R.L. Taylor, P.J. Beresford and E.L. Wilson, A nonconforming element for stress analysis, Internat. J. Numer. Methods Engrg. 10 (1976), 1211-1219.

[26] S.P. Timoshenko, On the correction for shear of the differential equation for transverse vibrations of prismatic bars, Philos. Mag. Ser 641 (1921), 744-746.

[27] E.L. Wilson, The static condensation algorithm, Internat. J. Numer. Methods Engrg. 8 (1974), 198-203.

[28] E.L. Wilson, R.L. Taylor, W.P. Doherty and J. Ghaboussi, Incompatible displacement models, In Numerical and Computer Methods in Structural Mechanics, (S.J. Fenves, P.N. Perrone, A.R. Robinson and W.C. Schnobrich eds.) 1973, pp. 43-57.

[29] M.J. Turner, R.W. Clough, H.C. Martin and L.J. Topp, Stiffness and deflection analysis of complex structures, J. Aeronaut. Sci. 23 (1956), 803-823, 854. 